\title{
Temperature effects on surface activity and application in oxidation of toluene derivatives of CTAB-SDS with $\mathrm{KMnO}_{4}$
}

\author{
YU TANG*, BIYING DU, JUN YANG and YUANMING ZHANG \\ Chemistry Department, Jinan University, Guangdong Guangzhou 510632, People's Republic of China \\ e-mail: ttangy123@yahoo.com.cn
}

MS received 9 September 2005; revised 11 November 2005

\begin{abstract}
The $\gamma_{\mathrm{cmc}}$ values of CTAB-SDS decrease from $63.67 \mathrm{mN} / \mathrm{m}$ at $10^{\circ} \mathrm{C}$ to $36.38 \mathrm{mN} / \mathrm{m}$ at $90^{\circ} \mathrm{C}$, slightly lower than those of either CTAB or SDS. Correspondingly, the CMC of CTAB-SDS decreases almost by half. The increase of surface activity of CTAB-SDS can be attributed to the relatively weak electrostatic interaction at high temperature, which is supported by the increase of solubility of CTABSDS with rise in temperature. Catalytic effect on oxidation of toluene derivatives with potassium permanganate follows the order CTAB-SDS > SDS > CTAB. This is not caused by the dissociative effect of CTAB-SDS with low surface activity at low temperature, as seen from the fact that almost all oxidative products can be retrieved for different toluene derivatives and surfactants by mimicking the conditions of reaction. In the emulsifications of toluene derivatives at $90^{\circ} \mathrm{C}$, the time that turbid water layers of surfactant solutions take to become clear is the same as that of the catalytic effect on oxidation of toluene derivatives. Thus, it can be inferred that surfactants can improve the oxidation yields of toluene derivatives by increasing the contact between two reacting phases.
\end{abstract}

Keywords. Surface activity; cetyl trimethylammonium bromide; sodium dodecyl sulfate; temperature; oxidation.

\section{Introduction}

Cationic systems show strong synergism in their solutions and display physicochemical properties that differ distinctly from those of individual surfactants, ${ }^{1}$ due to their electrostatic interaction between oppositely charged head groups, in addition to hydrophobic interaction. For instance, their high surface activity is good for emulsification because they reduce the oil-water interfacial tension. ${ }^{2}$ Moreover, a lamellar liquid crystalline phase is formed at some concentration ranges of cationic surfactants, but their equimolar mixtures invariably result in precipitates in water even at low concentration. ${ }^{3}$ The phase equilibria and the aggregation behaviors of several aqueous cationic systems have been described. ${ }^{4}$ Reports of cationic surfactants are also concerned about physico-chemical properties of CTAB/SDS at low temperatures ${ }^{5}$ and microemulsion formation with $\mathrm{CTAB} / \mathrm{SDS}^{6}{ }^{6}$

The effects of surfactant micelles on the kinetics of organic reactions have been investigated, ${ }^{7,8}$ because surfactant micelles can change the rate of chemical

\footnotetext{
*For correspondence
}

reactions that involves incorporation of reactants in the micellar pseudophase. ${ }^{9}$ Further, surfactants have also appeared in studies on organic reactions in microemulsions, ${ }^{10}$ organic syntheses, ${ }^{11}$ and the influence of reverse micelles on nucleophilic aromatic substitution reactions. ${ }^{12}$ Oxidations of oxalic acid by chromium(VI) ${ }^{13}$ and water-soluble colloidal $\mathrm{MnO}_{2}{ }^{14}$ are catalyzed by CTAB and TX-100 micelles, but not with SDS. Oxidation of some aminoalcohols with $\mathrm{N}$-bromosuccinimide in alkaline medium is accelerated by CTAB at low concentrations, ${ }^{15}$ while the oxidation of $\mathrm{D}(+)$-xylose with chromic acid in water is accelerated by SDS. ${ }^{16}$ Nowadays, potassium permanganate is extensively used as an oxidant. ${ }^{17,18}$ Trichloroethylene degradation by permanganate in aqueous solution can be speeded up in the presence of SDS. ${ }^{19}$ However, the effect of surfactant on oxidation by potassium permanganate has seldom been reported.

To the best of our knowledge, the relationship of surface activity of cationic surfactant with temperature has not been shown, and cationic surfactants have not been employed in organic reactions. In this work, surface tensions of equimolar CTAB-SDS at 
different temperatures have been determined and compared with those of corresponding individual surfactants. A reasonable explanation supported by solubility experiments has been proposed. Based on these results, equimolar CTAB-SDS has been used in oxidations of toluene derivatives by potassium permanganate. Its favorable role in obtaining oxidative yields of toluene derivatives has been investigated by recovering the products by mimicking the reaction conditions, and by emulsifying the toluene derivatives at $90^{\circ} \mathrm{C}$.

\section{Materials and methods}

All the chemicals used - potassium permanganate, sodium hydroxide, toluene derivates, aetyl trimethylammonium bromide (CTAB; Beijing Chemical Corp., purity $>99 \%$ ) and sodium dodecyl sulfate (SDS; Beijing Chemical Import Corp., purity $>99 \%$ ), were of analytical grade and used without further purification. Distilled water was used as solvent.

Surface tension measurement - Surface tensions $(\gamma)$ of aqueous solutions of CTAB, SDS and their equimolar mixture were measured by maximum bubble pressure tensiometry at different temperatures.

Solubility measurement - Aqueous solutions (10 ml) of both CTAB and SDS (surfactant concentrations are $0.005 \mathrm{M}$ ) were mixed and stirred at a certain temperature, then filtered through a cellulose membrane $(0.22 \mu \mathrm{m})$. A portion $(2 \mathrm{ml})$ of the clear filtrate was evaporated to dryness. Solubility of the equimolar mixture of CTAB and SDS at this temperature was calculated from the weight of the residue left behind.

Emulsification of toluene derivatives by surfactants - Equal volumes $(10 \mathrm{ml})$ of toluene derivatives and $0.01 \mathrm{M}$ aqueous surfactant solutions were mixed by thoroughly shaking five times by hand at $90^{\circ} \mathrm{C}$ in a measuring cylinder with a cap. The mixture was then kept aside at this temperature. The lapse of time that occurred before $6 \mathrm{ml}$ and $8 \mathrm{ml}$ of water layer separated, and also when the turbid water layers became clear was recorded.

Oxidation of toluene derivatives - Equal molar volumes of toluene derivatives (calculated by the methyl group), $2 \mathrm{~g}$ of $\mathrm{NaOH}$ and $100 \mathrm{ml}$ of $0.01 \mathrm{M}$ aqueous surfactant solutions were mixed and stirred.
When the mixture was heated to about $50^{\circ} \mathrm{C}$, three times the potassium permanganate with respect to the moles of toluene derivatives was added. The reaction was kept at a temperature above $90^{\circ} \mathrm{C}$ for $2 \mathrm{~h}$. After the mixture was cooled and filtered, dilute sulfuric acid was added to adjust the solution $\mathrm{pH}$ to 2 . The precipitate was filtered and washed with distilled water. The structures of the products were confirmed by measuring their m.p., IR and ${ }^{1} \mathrm{H}$ NMR spectra.

\section{Results and discussion}

\subsection{Effects of temperature on surface activities of surfactants}

Values of surface tensions of surfactants vs concentrations were measured at different temperatures (figures 1,2 , and 3 ). The results indicate that the surface tension of surfactants decreases with increase in temperature.. The decrease of $\gamma_{\mathrm{cmc}}$ is very small for both CTAB and SDS (table 1), hence their surface tensions were measured at $20^{\circ} \mathrm{C}$ intervals. The $\gamma_{\mathrm{cmc}}$ of their equimolar mixture however decreases from $63.67 \mathrm{mN} / \mathrm{m}$ at $10^{\circ} \mathrm{C}$ to $36.38 \mathrm{mN} / \mathrm{m}$ at $90^{\circ} \mathrm{C}$, a slightly lower value than that of either $\mathrm{CTAB}$ or SDS. Correspondingly, the CMC of CTAB and SDS decreases slightly, while the CMC of their equimolar mixture decreases almost by half. Thus, it can be concluded that the surface activity of CTAB-SDS mixture increases to a large extent with temperature increase, and is slightly higher than that of CTAB or SDS at high temperature $\left(90^{\circ} \mathrm{C}\right)$.

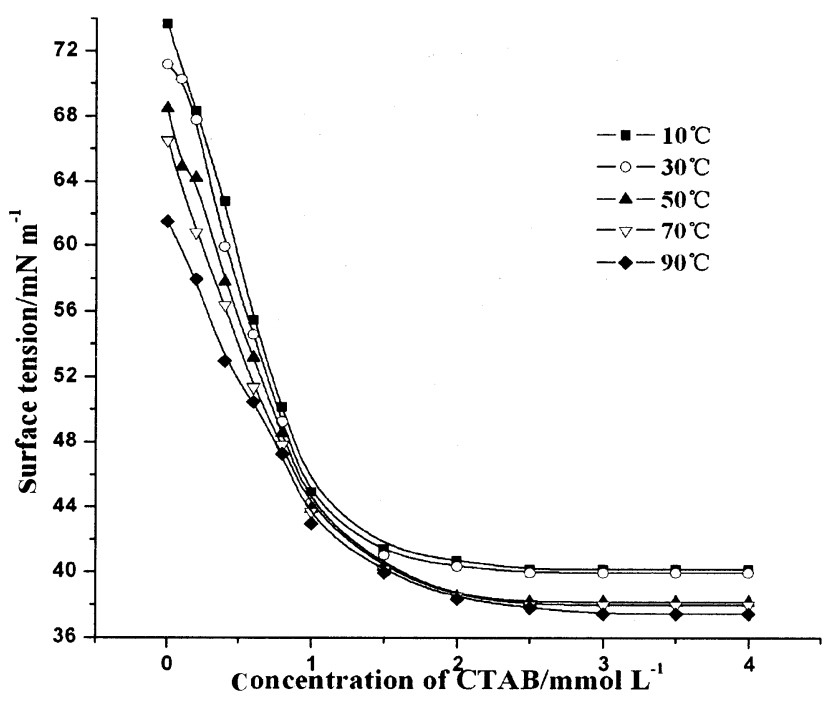

Figure 1. Surface tension of CTAB at different temperatures. 
Table 1. CMC and $\gamma_{\mathrm{cmc}}$ of CTAB, SDS and CTAB-SDS at different temperatures.

\begin{tabular}{lccccccc}
\hline & \multicolumn{3}{c}{$\mathrm{CMC}\left(\mathrm{mmol}^{-1}\right)$} & & \multicolumn{3}{c}{$\gamma_{\mathrm{cmc}}\left(\mathrm{mN} \mathrm{m}^{-1}\right)$} \\
\cline { 2 - 3 } \cline { 7 - 8 } Temp $\left({ }^{\circ} \mathrm{C}\right)$ & CTAB-SDS & CTAB & SDS & & CTAB-SDS & CTAB & SDS \\
\hline 10 & 4.80 & 1.14 & 4.10 & & 63.67 & 42.45 & 42.98 \\
20 & 4.65 & & & & 60.99 & & \\
30 & 4.55 & 1.12 & 4.08 & & 57.25 & 39.94 & 40.13 \\
40 & 3.30 & & & & 52.97 & & \\
50 & 2.90 & 1.10 & 4.05 & & 50.07 & 38.17 & 39.59 \\
60 & 2.80 & & & & 48.33 & & \\
70 & 2.72 & 1.09 & 4.02 & & 45.09 & 37.99 & 39.24 \\
80 & 2.68 & & & & 42.73 & & \\
90 & 2.65 & 1.07 & 4.00 & & 36.38 & 37.45 & 37.63 \\
\hline
\end{tabular}

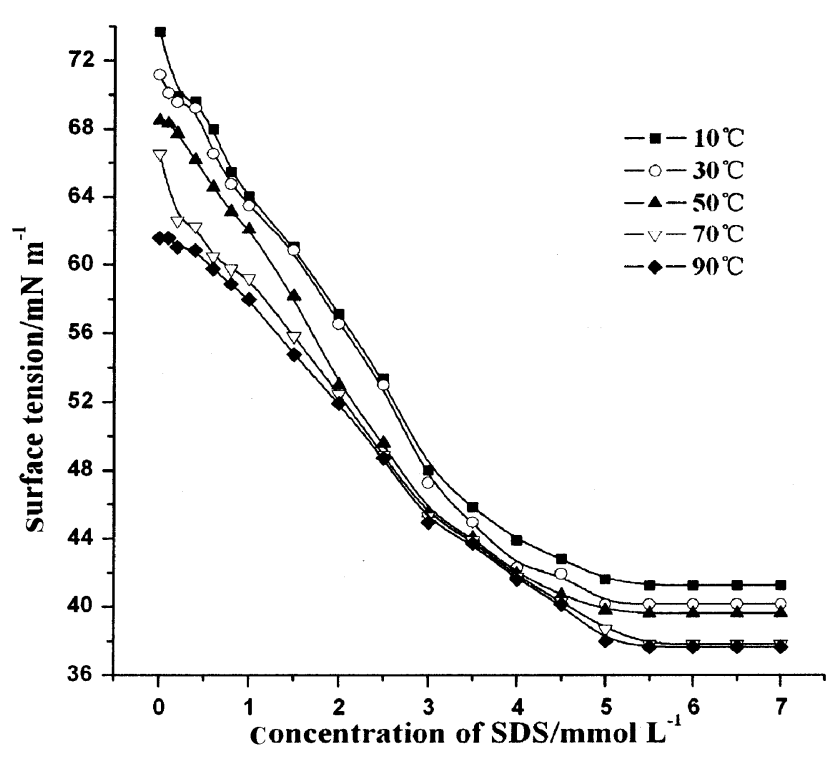

Figure 2. Surface tension of SDS at different temperatures.

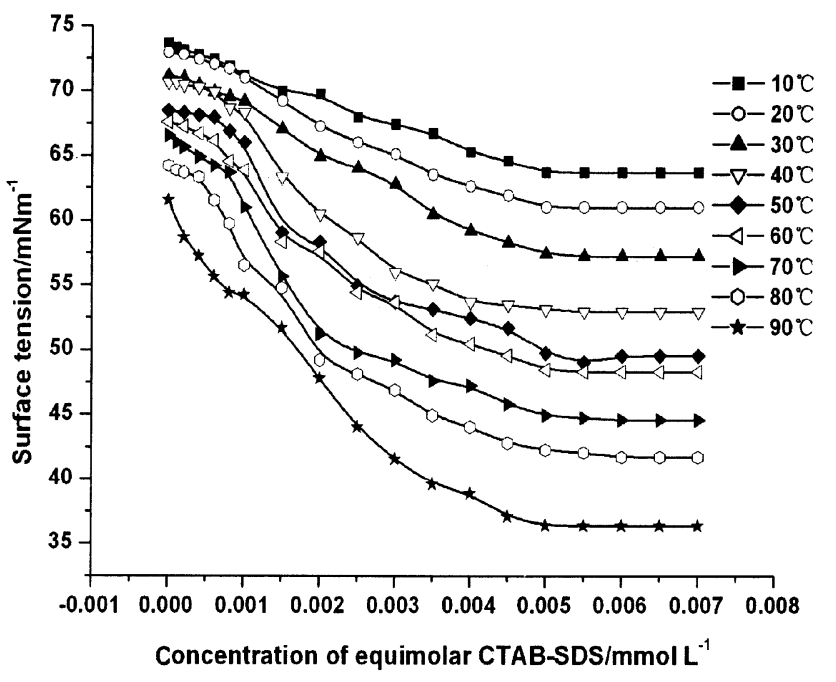

Figure 3. Surface tension of CTAB-SDS at different temperatures.
Anionic and cationic surfactants precipitate and cause a decrease in their surface activity when they are both present in water due to their strong electrostatic interaction. However, even the equimolar mixture of CTAB and SDS shows surfactant character at a fairly low temperature $\left(10^{\circ} \mathrm{C}\right)$ (figure 3$)$. As temperature increases, the large decreasing surface tension of this mixture can be attributed to the fact that electrostatic interaction becomes weak. ${ }^{20}$

\subsection{The solubility of CTAB-SDS}

If the weak electrostatic interaction at high temperature can cause the increase of surface activity of the CTAB-SDS mixture, its solubility should also increase with increase in temperature. This assumption is right as shown in table 2 . The solubility at $90^{\circ} \mathrm{C}$, though still a low value, is more than two times compared with that at $10^{\circ} \mathrm{C}$. From $10^{\circ} \mathrm{C}$ to $40^{\circ} \mathrm{C}$, the low temperature range, although the values of solubility are smaller than the CMC at the same temperature, the CTAB-SDS mixture still shows the characteristics of typical surfactant. That is to say, the insoluble CTAB-SDS can decrease the surface tension of the solution also. This characteristic makes the cationic system a possible application in some solid situation.

\subsection{Oxidation of toluene derivatives}

The high surface activity of CTAB-SDS mixture is favored for reducing the interface tension of heterogeneous organic reactions at high temperature, while its low surface activity and solubility would help to separate the product and recover the surfactants at low temperature. Hence, CTAB-SDS was employed in the oxidation of toluene derivatives with potassium permanganate. 
Table 2. Solubilities of CTAB-SDS at different temperatures.

\begin{tabular}{lccccccccc}
\hline Temperature $\left({ }^{\circ} \mathrm{C}\right)$ & 10 & 20 & 30 & 40 & 50 & 60 & 70 & 80 & 90 \\
Solubility $\left(\mathrm{mmol} \mathrm{l} \mathrm{l}^{-1}\right)$ & 1.84 & 1.88 & 1.99 & 2.76 & 3.68 & 3.91 & 4.03 & 4.29 & 4.56 \\
\hline
\end{tabular}

Table 3. Effect of CTAB, SDS and CTAB-SDS on the oxidation of toluene derivatives.

\begin{tabular}{|c|c|c|c|c|c|c|}
\hline \multirow[b]{2}{*}{ Reactants } & \multicolumn{2}{|c|}{ CTAB } & \multicolumn{2}{|c|}{ SDS } & \multicolumn{2}{|c|}{ CTAB-SDS } \\
\hline & Yield (\%) & Ret. yield* (\%) & Yield (\%) & Ret. yield* (\%) & Yield (\%) & Ret. yield* (\%) \\
\hline Toluene & $23 \cdot 5$ & $94 \cdot 5$ & $26 \cdot 5$ & $94 \cdot 5$ & $49 \cdot 2$ & $98 \cdot 5$ \\
\hline$p$-Nitrotoluene & $38 \cdot 5$ & $93 \cdot 0$ & $42 \cdot 3$ & $95 \cdot 0$ & $49 \cdot 7$ & $96 \cdot 0$ \\
\hline Benzyl chloride & $51 \cdot 9$ & $94 \cdot 5$ & $57 \cdot 5$ & $94 \cdot 5$ & $60 \cdot 3$ & $98 \cdot 5$ \\
\hline$p$-Xylene & $64 \cdot 8$ & $98 \cdot 5$ & $70 \cdot 0$ & $98 \cdot 5$ & $78 \cdot 8$ & $99 \cdot 0$ \\
\hline
\end{tabular}

*Ret. yield = retrievable yield

Table 4. Emulsifications of toluene derivatives at $90^{\circ} \mathrm{C}$.

\begin{tabular}{|c|c|c|c|c|c|c|c|c|c|}
\hline \multirow[b]{2}{*}{ Reactants } & \multicolumn{3}{|c|}{ Time $(\min )^{\mathrm{a}}$} & \multicolumn{3}{|c|}{ Time $(\min )^{b}$} & \multicolumn{3}{|c|}{ Time $(\min )^{\mathrm{c}}$} \\
\hline & CTAB-SDS & SDS & СТАВ & CTAB-SDS & SDS & СТАВ & CTAB-SDS & SDS & СТАВ \\
\hline Toluene & $11 \cdot 30$ & $3 \cdot 82$ & $9 \cdot 42$ & $32 \cdot 06$ & $28 \cdot 86$ & 31.79 & $>480$ & $130 \cdot 00$ & $100 \cdot 00$ \\
\hline$p$-Nitrotoluene & 0.69 & $1 \cdot 80$ & $7 \cdot 57$ & 1.07 & $5 \cdot 44$ & $28 \cdot 05$ & $110 \cdot 00$ & $38 \cdot 00$ & $30 \cdot 00$ \\
\hline Benzyl chloride & $9 \cdot 80$ & $13 \cdot 83$ & $22 \cdot 68$ & $12 \cdot 59$ & $15 \cdot 09$ & $25 \cdot 14$ & $35 \cdot 00$ & $24 \cdot 50$ & $11 \cdot 00$ \\
\hline$p$-Xylene & $55 \cdot 00$ & $4 \cdot 41$ & $5 \cdot 98$ & $250 \cdot 00$ & $8 \cdot 09$ & $19 \cdot 27$ & $>480$ & $150 \cdot 00$ & $70 \cdot 00$ \\
\hline
\end{tabular}

${ }^{\mathrm{a}}$ For the time period when $6 \mathrm{ml}$ water was separated, ${ }^{\mathrm{b}}$ for the time period when $8 \mathrm{ml}$ water was separated, ${ }^{\mathrm{c}}$ for the time period till aqueous layer became clear

The results indicate that the oxidation yields of toluene derivatives catalyzed by CTAB-SDS system are higher than those catalyzed by CTAB and SDS (table 3). The order of the catalytic effect on oxidation of toluene derivatives, $\mathrm{CTAB}-\mathrm{SDS}>\mathrm{SDS}>\mathrm{CTAB}$, can be inferred from the yields in table 3 . The oxidative products of toluene derivatives were dissolved and recovered by mimicking the conditions of reaction to see if the higher yields were owing to the good separation role of CTAB-SDS at low temperature. The natural conditions were as follows: $2 \mathrm{~g}$ of product, $2 \mathrm{~g}$ of $\mathrm{NaOH}$ and $100 \mathrm{ml}$ of $0.01 \mathrm{M}$ aqueous surfactant solutions were mixed, stirred and heated at above $90^{\circ} \mathrm{C}$ for half an hour. Then the mixture was cooled and filtered, and dilute sulphuric acid was added to adjust the solution $\mathrm{pH}$ to 2 . The precipitate was filtered, washed with distilled water and dried, and the retrievable yields were calculated from the weight of the precipitate. Almost all products have been obtained for different toluene derivatives and surfactants. Thus there must be some other effects of CTAB-SDS.

\subsection{Emulsification of surfactants}

The emulsifications of toluene derivatives at the reaction temperature $90^{\circ} \mathrm{C}$ were carried out to investigate the effect of surface activity of different surfactants on the heterogeneous reactions (table 4).

Although the separating time of water layer is a sign of emulsion stability, no obvious rule existing in the results can explain the order of oxidative yields of toluene derivatives catalyzed by surfactants. Although the contact of two phases facilitated by surfactant is helpful for the stability of emulsion, the key factor for separating two phases of emulsion is the strength of interface membrane. It is interesting to note that the order of the times when aqueous layers of surfactant solutions become clear is CTAB-SDS > SDS > CTAB, the same as that of the catalytic effect on oxidation of toluene derivatives. The turbid aqueous solution is caused by slight toluene derivatives emulsified in water. Obviously, the longer time the turbid water takes to become clear, the more the two phases can stay contact. Thus, the surfactants 
Table 5. Oxidation yields of toluene under different concentrations of surfactants.

\begin{tabular}{lccc}
\hline$C\left(\mathrm{~mol} \mathrm{l}^{-1}\right)$ & $Y_{\mathrm{CTAB}-\mathrm{SDS}}(\%)$ & $Y_{\mathrm{SDS}}(\%)$ & $Y_{\text {СТАВ }}(\%)$ \\
\hline 0.005 & $43 \cdot 2$ & $22 \cdot 5$ & $20 \cdot 8$ \\
0.01 & $49 \cdot 2$ & 26.5 & 23.5 \\
0.03 & 52.5 & 28.4 & $25 \cdot 7$ \\
0.06 & 54.1 & $30 \cdot 2$ & 27.3 \\
\hline
\end{tabular}

can improve the oxidation yields of toluene derivatives through facilitating the contact of two reaction phases. If this is a fact, better oxidation yields of toluene derivatives can be given when catalyzed by higher concentrations of surfactants. For this reason, toluene was oxidized under different concentrations of surfactants. Although the increase of oxidative yields between two neighboring concentrations of surfactants is small, the trend that oxidative yields of toluene increase with the increment of concentrations of surfactants is clear (table 5). Unfortunately, our effort to get a much better oxidative yield of toluene at a higher concentration of surfactant than $0 \cdot 1 \mathrm{~mol} / \mathrm{l}$ was unsuccessful because too much of foam occurred in the process of reaction and filtration was difficult after the reaction. Even so, the conclusion mentioned above can be supported by these results.

\section{Conclusion}

In summary, the following conclusions can be drawn. With the increase of temperature, the increase of surface activity of CTAB and SDS is very small. The surface activity of CTAB-SDS mixture greatly increases with temperature increase, and is slightly higher than that of CTAB or SDS at high temperature $90^{\circ} \mathrm{C}$. It can be attributed to the changed weak electrostatic interaction. This is supported by the result that the solubility of equimolar CTAB-SDS mixture increases with increase in temperature. The contact of toluene derivatives with water can be improved by CTAB-SDS. This gives the reasonable explanation of better oxidative yields of toluene derivatives catalyzed by CTAB-SDS than by CTAB and SDS.

\section{Acknowledgement}

This work was supported by Natural Science Group Fund of Guangdong Province (039213).

\section{References}

1. Zhu B Y and Rosen M J 1984 J. Colloid Interface Sci. 99435

2. Walstra P 1993 Chem. Eng. Sci. 48333

3. Jönsson B, Jokela A, Khan B, Lindman B and Sadaghiani A 1991 Langmuir 7889

4. Caria A and Khan A 1996 Langmuir 126282

5. Jiang P C and Lu J 1997 J. Shanghai Teachers Univ. (Nat. Sci.) 2640

6. Li X F, Lin E H, Zhao G H and Xiao T 1996 J. Colloid Interface Sci. $\mathbf{1 8 4} 20$

7. Bhattacharya S and Kumar V P 2005 Langmuir 21 71

8. Zakharova L, Valeeva F, Zakharov A, Ibragimova A, Kudryavtseva L and Harlampidi H 2003 J. Colloid Interface Sci. 263597

9. Svensson R, Pamedytyte V, Juodaityte J, Makuška R and Morgenstern R 2001 Toxicology 168251

10. Häger M, Currie F and Holmberg K 2004 Colloid Surf. A250 163

11. Krafft M E, Wright J A and Bonäga L V R 2003 Tetrahedron Lett. 443417

12. Correa N M, Durantini E N and Silber J J 2000 J. Org. Chem. 656427

13. Kabir-ud-Din, Hartani K and Khan Z 2001 Colloid Surf. A193 1

14. Kabir-ud-Din, Fatma W and Khan Z 2004 Colloid Surf. A234 159

15. Pandey S and Upadhyay S K 2005 J. Colloid Interface Sci. 285789

16. Kabir-Ud-Din, Morshed A A and Khan Z 2001 Inorg. React. Mech. 3255

17. Hajipour A R, Adibi H and Ruoho A E 2003 J. Org. Chem. 684553

18. Shaabani A, Mirzaei P, Naderia S and Lee D G 2004 Tetrahedron 6011415

19. Li Z H 2004 Chemosphere 54419

20. Gong Y J and Xue Y Y 2000 J. Northwest Univ. (Nat. Sci. Edn) 130 